\title{
A proteomic approach to the identification of the major virion structural proteins of the marine cyanomyovirus S-PM2
}

\begin{abstract}
Correspondence
Martha R. J. Clokie

mrjc1@le.ac.uk
\end{abstract}

Received 26 December 2007

Revised 5 March 2008

Accepted 12 March 2008

\author{
Martha R. J. Clokie, ${ }^{1} \dagger$ Konstantinos Thalassinos, ${ }^{2} \dagger$ Pascale Boulanger, ${ }^{3}$ \\ Susan E. Slade, ${ }^{2}$ Svetla Stoilova-McPhie, ${ }^{2}$ Matt Cane, ${ }^{2}$ James H. Scrivens ${ }^{2}$ \\ and Nicholas H. Mann² \\ ${ }^{1}$ Department of Infection, Immunity and Inflammation, Maurice Shock Medical Sciences Building,
University of Leicester, Leicester LE1 9HN, UK
${ }^{2}$ Department of Biological Sciences, University of Warwick, Coventry CV4 7AL, UK
${ }^{3} \mathrm{U}$ IBBMC-CNRS UMR 8619, Bât. 430 - Université de Paris-Sud, F-91405 Orsay, Paris, France
}

In this study, an MS-based proteomics approach to characterizing the virion structural proteins of the novel marine 'photosynthetic' phage S-PM2 is presented. The virus infects ecologically important cyanobacteria of the genus Synechococcus that make a substantial contribution to primary production in the oceans. The S-PM2 genome encodes $236 \mathrm{ORFs}$, some of which exhibit similarity to known phage virion structural proteins, but the majority $(54 \%)$ show no detectable homology to known proteins from other organisms. Using public and in-house bioinformatics tools the proteome of S-PM2 was predicted and a database compatible with MS-based search engines was constructed. S-PM2 virion proteins were resolved by SDS-PAGE, excised, tryptically digested and analysed by LC-ESI-MS/MS. The resulting MS data were searched against the database. A parallel control study was undertaken on the well-characterized coliphage T4 in order to assess the sensitivity and efficiency of this approach. In total, 11 of the 15 S-PM2 proteins, predicted to be virion proteins by bioinformatics approaches, were confirmed as such, together with the identification of a further 12 novel structural proteins. In the case of T4, 24 of the 39 known virion structural proteins were identified, including the major tail-fibre proteins. This approach has wide-ranging applicability and can be applied to any novel organism whose genome encodes ORFs with few detectable homologies in the public databases.

\section{INTRODUCTION}

Interest in the study of bacteriophages has increased dramatically in recent years, largely as a result of the growing recognition of their ecological importance (Fuhrman, 1999; Suttle, 2007). Consequently, viruses infecting bacteria and archaea are currently being isolated and characterized. Genomic and transcriptomic studies are revealing novel features of the interactions between phages and their hosts that significantly expand the paradigms derived from the study of the classical phage-host systems. One area where the study of these novel phages is not progressing so rapidly is their structural biology. Genomic data can often be used to identify structural components of the virion on the basis of homology, but this approach is usually restricted in its success. An example of this can be seen in genomes of phages that infect archaea where many phage genomes contain no ORFs with similarity to known proteins (Prangishvili \& Garrett, 2004). Thus, while it is

†These authors contributed equally to this work. very useful to have whole-genome sequences for phages, additional characterization is often necessary. To establish which ORFs in novel phage genomes encode structural proteins, an MS-based proteomics and bioinformatics approach was undertaken.

The phage selected for this study was S-PM2 which infects marine cyanobacteria of the genus Synechococcus. These organisms, together with their close relatives from the genus Prochlorococcus, make a substantial contribution to the primary productivity of the oceans (Field et al., 1998). This phage was originally isolated from water collected off the coast of Plymouth, UK (Wilson et al., 1993). It is a lytic myovirus with an icosahedral head and contractile tail and its genome has been sequenced (Mann et al., 2005).

The archetypal myovirus T4 devotes more than $40 \%$ of its genome to the synthesis and assembly of one of the most complex virus particles known (Miller et al., 2003a). SPM2 is morphologically similar to T4 and therefore it is likely that a similar number of proteins are involved in 
building its virion structure. The genome of S-PM2 is slightly larger than T4 and contains 236 ORFs, 69 of which exhibit similarity to other phage or bacterial genes of known function, including 38 which have similarity to T4 (Mann et al., 2005), 15 of which have similarity to hypothetical proteins and the remaining 152 have no similarity to any known proteins.

There is a surprising degree of synteny between the S-PM2 and T4 ORFs which encode virion structural genes (Hambly et al., 2001; Mann et al., 2005). It is known that the T4 virion is composed of around 39 structural proteins (Miller et al., 2003a), 15 of which have homologues in SPM2. These ORFs are shown in Fig. 1 and appear to comprise a core set of conserved proteins that have not evolved significantly from an ancestral T4-type phage. These form the complex contractile tail necessary for the injection of phage DNA (Fig. 1). Both phages encode the major capsid protein (gp23), three neck proteins (gp20, gp13 and gp14), four tail proteins (gp3, gp15, gp18 and gp19) and six baseplate hub proteins that are part of the baseplate-tail tube complex (gp5, gp6, gp8, gp25, gp48 and gp53) (Kostyuchenko et al., 2003). This leaves in the order of 20 S-PM2 virion structural proteins to be identified, which was the purpose of this study. T4 was used as a control to assess the efficiency of the proteomic approach developed in this study.

\section{METHODS}

Cyanobacteria, phage and media. Synechococcus sp. WH7803 was used to propagate S-PM2. It was grown in artificial seawater (ASW) as described by Wilson et al. (1993). To harvest phage, exponentially growing 11 Synechococcus cultures $\left(\mathrm{OD}_{750}\right.$ between 3.5 and 4$)$ were lysed to give a phage titre with an m.o.i. of approximately 1. Lysed cultures were treated with $4 \mathrm{ml}$ chloroform $1^{-1}$ and $10 \mu \mathrm{g}$ DNase I $\mathrm{ml}^{-1}$ for $3 \mathrm{~h}$. Cell debris was then removed with $10 \% \mathrm{NaCl}$ followed by centrifugation at $8000 \mathrm{~g}$ in a Beckman centrifuge (Beckman Coulter) for $10 \mathrm{~min}$. Phage were precipitated with PEG 6000 (Sigma) and collected by centrifugation at $10000 \mathrm{~g}$ for $10 \mathrm{~min}$ at $4{ }^{\circ} \mathrm{C}$. The $\mathrm{CsCl}$ step and equilibrium gradients were prepared as described by Sambrook et al. (1989).

Phage preparation. Phage were removed from the $\mathrm{CsCl}$ gradients and dialysed in SM buffer $\left(\mathrm{l}^{-1}: 5.8 \mathrm{~g} \mathrm{NaCl}, 2 \mathrm{~g} \mathrm{MgSO}_{4} \cdot 7 \mathrm{H}_{2} \mathrm{O}, 50 \mathrm{ml}\right.$ $1 \mathrm{M}$ Tris/HCl, pH 7.5). Phage ghosts were prepared as described by Konopa \& Taylor (1979). Briefly, phages were concentrated by ultracentrifugation at $100000 \mathrm{~g}$ in a TL100 (Beckman Coulter) at $4{ }^{\circ} \mathrm{C}$ for $30 \mathrm{~min}$. They were resuspended in $10 \mathrm{M} \mathrm{LiCl}$ and heated to $46{ }^{\circ} \mathrm{C}$ for $20 \mathrm{~min}$, then diluted $10 \times$ in $50 \mathrm{mM}$ Tris $/ \mathrm{HCl}, \mathrm{pH} 8$, $100 \mathrm{mM} \mathrm{NaCl}$ and $5 \mathrm{mM} \mathrm{MgCl}_{2}$, and treated with 40 units DNase (Ambion) for $2 \mathrm{~h}$ at $37^{\circ} \mathrm{C}$. Samples were then reconcentrated and resuspended in protein buffer, boiled for $2 \mathrm{~min}$ and then spun down to remove any particulate matter.

Protein preparation and identification. The supernatant from above was resolved on a 1-D SDS-PAGE gel which was stained with $0.1 \%$ Coomassie brilliant blue G-250 (Bio-Rad) in $40 \%$ methanol and $10 \%$ acetic acid for $1 \mathrm{~h}$. The gel was destained in $40 \%$ methanol and $10 \%$ acetic acid until the background had faded (after around $2 \mathrm{~h}$ ) and stored in deionized water until required.

Protein digestion. Protein gel plugs were excised and processed automatically by the MassPrep robotic protein handling system (Waters Micromass MS Technologies), using the manufacturer's recommended protocol. In brief, the gel plugs were destained, the disulfide bonds were reduced by the addition of dithiothreitol and the free cysteine residues were alkylated with iodoacetamide. The gel plugs were washed prior to a dehydration step, followed by the addition of trypsin, and incubated for $4.5 \mathrm{~h}$. The resultant tryptic peptides were extracted up to three times in total and transferred to a cooled 96-well microtitre plate; if necessary, they were stored at $-20{ }^{\circ} \mathrm{C}$.

Analysis by liquid chromatography electrospray ionization tandem MS (LC-ESI-MS/MS). The 96-well microtitre plate containing the extracted tryptic peptides was transferred to a modular

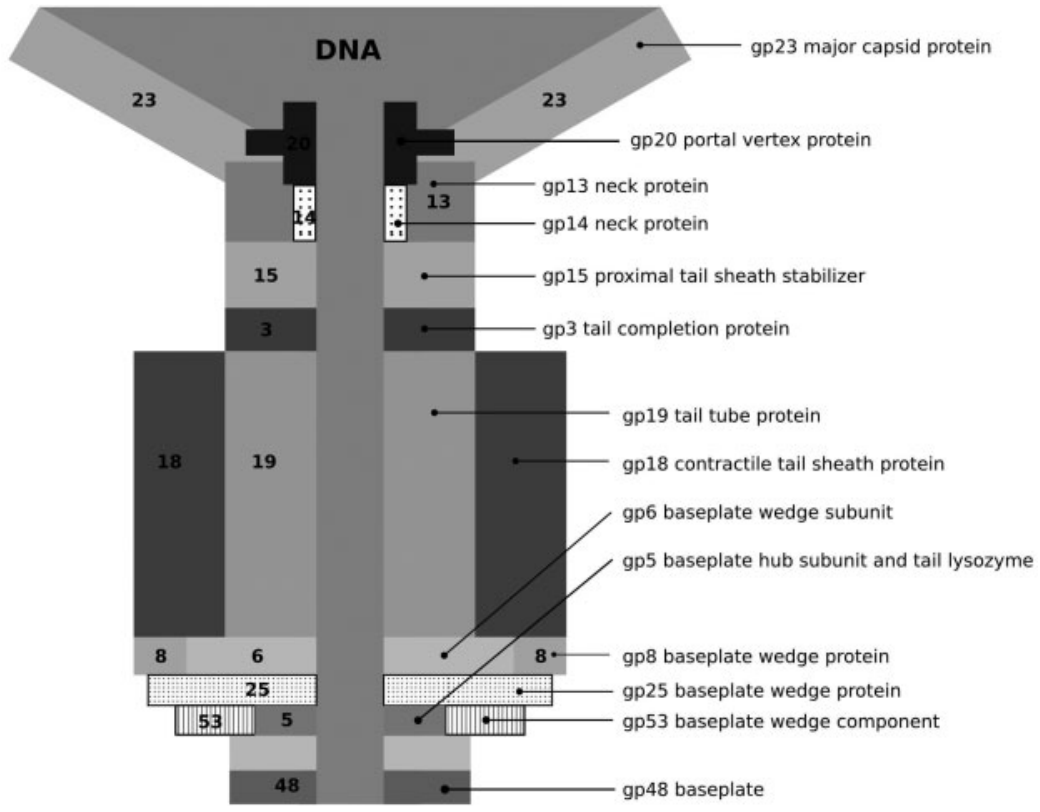

Fig. 1. Prediction of the organizational relationship of the core set of mature structural proteins conserved between S-PM2 and T4, drawn using information from Miller et al. (2003a) and Leiman et al. (2004). Ten of these predicted 14 homologues were detected in our study. We also detected the gp22 (not shown here as it is not present in the mature capsid). Proteins not detected were gp14, gp25, gp53 and gp5. 
CapLC and autosampler system (Waters Micromass MS Technologies). A $5 \mu \mathrm{l}$ aliquot of the extract was mixed with $15 \mu \mathrm{l}$ $0.1 \%$ formic acid and loaded onto an LC Packings $\mu$-Precolumn (C18 PepMap 100, $5 \mu \mathrm{m}, 100 \AA$, $300 \mu \mathrm{m}$ i.d. $\times 5$ mm) cartridge. Flushing the column with solution A desalted the bound peptides before a linear gradient of solution $\mathrm{B}$ at a flow rate of $0.2 \mathrm{nl} \mathrm{min}^{-1}$ eluted the peptides for further resolution on a $15 \mathrm{~cm}$ LC Packings C18 PepMap analytical column $(5 \mu \mathrm{m}, 5 \AA$, $75 \mu \mathrm{m}$ i.d. $)$.

ProteinLynx Global Server 2.0 (Waters Micromass MS Technologies) was used to interrogate the data obtained from the LC-ESI-MS/MS experiments. MS/MS protein identifications were accepted with a minimum of one manually validated peptide per protein.

Construction of the protein database. Identification of a protein by MS-based proteomics approaches requires the proteins of the organism under investigation (or a close homologue) to be present in a database. At the time the project was conducted, S-PM2 and the host Synechococcus WH7803 had only recently been sequenced and the genomes were not present in any existing databases. The genomic sequence of S-PM2 was used to predict potential protein sequences which were then added to the latest release of the SWISS-PROT database. This allowed identification of contaminants both in the form of host proteins and those introduced during sample handling (mainly keratins). The use of a large database provided higher confidence in the database interrogation results; if the database consisted only of S-PM2 proteins, false-positives would be more likely. The raw genomic sequence of S-PM2 and Synechococcus WH7803 were translated in all six reading frames using the Translate tool from the ExPASy web server (http://ca.expasy.org/). ORFs giving rise to proteins with a molecular mass greater than $3000 \mathrm{Da}$ were included in the database. Genes were also predicted using GeneMarkS (http://opal.biology.gatech.edu/GeneMark/) and Glimmer (www. tigr.org/software/glimmer/). The output was converted to a FASTAformatted database by the use of Perl scripts, and all three databases were added to SWISS-PROT. The different methods of creating a database allowed for all possible proteins to be identified.

Cryoelectron microscopy. A sample $(5 \mu \mathrm{l})$ of an S-PM2 phage suspension was deposited on a pre-cleaned carbon-coated lacey grid (Agar Ltd). After 1 min incubation, the excess liquid was blotted and the grids were flash-frozen in liquid ethane cooled by liquid nitrogen. In this way the phages were trapped in a thin layer of amorphous ice in as close to native conditions as possible (Frank, 2006).

Digital images were collected at $\times 70000$ magnification on a $4096 \times 4096$ pixel $(15 \mu \mathrm{m}$ per pixel resolution) CCD camera (Ultrascan 4000; Gatan Inc.) with a JEM2010-FEG transmission electron microscope operated at $200 \mathrm{kV}$ and at low-dose conditions $\left(<16\right.$ electrons $\left.\AA^{-2}\right)$. All images were visualized and evaluated with Digital Micrograph Software (Gatan Inc.).

\section{RESULTS AND DISCUSSION}

The structural proteins of CsCl-gradient-purified S-PM2 and $\mathrm{T} 4$ virions were resolved by 1-D SDS-PAGE gels. The bands were manually excised, processed and subjected to tryptic digestion. The tryptic fragments were analysed using nano-LC-ESI-MS/MS. The resulting data consisted of peptide and peptide fragment masses and were used to interrogate a protein database that included S-PM2 and T4 protein sequences. This method has several advantages over an N-terminal sequencing approach; it is much faster and significantly fewer sample quantities are required. In addition, the approach allows the analysis of mixed proteins.

\section{Database interrogation}

A list of the number of matched peptides per protein for both T4 and S-PM2 is shown in Table 1. For S-PM2, all ORF numbers are consistent with the latest version of the genome published on EMBL website (www.ebi.ac.uk/embl/). The table also displays the molecular mass of identified proteins and the copy number for T4 proteins (if known). All proteins identified were predicted by both the GeneMark and Glimmer gene prediction programs.

\section{Cryoelectron microscopy}

The cryoelectron images of S-PM2 reveal the phage in its extended (Fig. 2a, b, d) and contracted form (Fig. 2c). The striations on the tail sheaths are quite clear, as are the long and short tail fibres and baseplate. In addition, Fig. 2(b, c) show empty phage capsids. One can see quite clearly that, unlike T4, there are no apparent whiskers at the top of the neck.

\section{Proteins identified}

This approach allowed the identification of 24 of 39 T4 virion structural proteins (Table 1). These were: 5 of the 8 head proteins (gpalt, gp23, gp24, gphoc and internal protein III), 12 of the 18 tail and baseplate proteins (gp5, gp6, gp7, gp8, gp9, gp10, gp11, gp15, gp18, gp19, gp27 and gp48), 3 of 4 proteins from the neck region (gp13, gp20 and gpwac), and 4 of the 5 tail-fibre proteins (gp12, gp34, gp36 and gp37). All proteins identified in T4 have been demonstrated to be part of the phage particle (Miller et al., 2003a). In general the T4 proteins identified are present in the capsid in high copy number (Table 1) but this is not universally the case as we were unsuccessful in detecting some proteins that were present with a high copy number, but we did manage to detect some with a low copy number.

When the same approach was applied to S-PM2, 23 structural proteins were identified (Table 1). There was a close match between the position of the protein on the gel and the predicted molecular mass of the protein (Fig. 3a). ORFs encoding 11 of the 15 predicted virion structural proteins (Mann et al., 2005), were confirmed as such. These were the major capsid protein gp23, the prohead assembly protein gp22, 2 of the 3 neck proteins (gp13 and gp20), and 7 of the tail and baseplate proteins (gp3, gp6, gp8, gp15, gp18, gp19 and gp48). A further 12 ORFs were established to encode S-PM2 virion structural proteins, which have no detectable homology to any entries in the public protein databases (Table 1).

\section{Head/whiskers/neck}

The most abundant protein in the T4 virion is the major capsid protein gp 23 and thus it is not surprising that it was identified in both T4 and S-PM2 with such high coverage (14 and 22 peptides respectively). The presence in S-PM2 of gp22 was interesting as this protein is part of a prohead that is degraded after the major capsid has formed (Black $e t$ 
Table 1. The number of matched peptides per protein for both T4 and S-PM2

For S-PM2, all ORF numbers are consistent with the latest version of the genome published on the EMBL website (www.ebi.ac.uk/embl/). The table also displays the molecular mass of identified proteins and the copy number for T4 proteins (if known).

\begin{tabular}{|c|c|c|c|c|c|c|c|c|}
\hline \multirow[t]{2}{*}{$\begin{array}{l}\text { T4 gene } \\
\text { product }\end{array}$} & \multirow[t]{2}{*}{ Function } & \multicolumn{2}{|c|}{ No. of peptides } & \multicolumn{2}{|c|}{ Copy no.* } & \multirow[t]{2}{*}{ S-PM2 ORF no. } & \multicolumn{2}{|c|}{$\begin{array}{c}\text { Molecular mass } \\
\text { (Da) }\end{array}$} \\
\hline & & T4 & S-PM2 & Precursor & Mature & & T4 & S-PM2 \\
\hline \multicolumn{9}{|l|}{ Head } \\
\hline gpalt & Phage head compontent & 8 & - & 40 & 40 & - & 75817 & - \\
\hline gp23 & Major capsid protein & 14 & 22 & 960 & 960 & 108 & 56021 & 50458 \\
\hline gp24 & Head vertex protein & 8 & - & 55 & 55 & - & 46993 & - \\
\hline gphoc & Head outer capsid protein & 3 & - & 0 & 160 & - & 40387 & - \\
\hline IP I & Internal head protein & - & - & 360 & 360 & - & - & - \\
\hline IP II & Internal head protein & - & - & 360 & 360 & - & - & - \\
\hline IP III & Internal head protein & 4 & - & 370 & 370 & - & 21687 & - \\
\hline gpsoc & Small outer capsid protein & - & - & 0 & 840 & - & - & - \\
\hline \multicolumn{9}{|l|}{ Prohead } \\
\hline gp21 & $\begin{array}{l}\text { Prohead core scaffold protein and } \\
\text { protease }\end{array}$ & - & - & 72 & 3 & - & - & - \\
\hline gp22 & Prohead core protein & - & 3 & 576 & 115 & 107 & - & 42473 \\
\hline gp67 & $\begin{array}{l}\text { Prohead core protein, precursor to } \\
\text { internal peptides }\end{array}$ & - & - & 341 & 134 & - & - & - \\
\hline gp68 & Prohead core protein & - & - & 240 & 0 & - & - & - \\
\hline \multicolumn{9}{|l|}{ Neck } \\
\hline gpwac & Collar protein & 9 & - & - & 18 & - & 51871 & - \\
\hline gp13 & Neck protein & 1 & 5 & - & 10 & 93 & 34741 & 32316 \\
\hline gp14 & Neck protein & - & - & - & 5 & - & - & - \\
\hline gp20 & Portal vertex protein & 5 & 5 & 12 & 12 & 104 & 61032 & 65363 \\
\hline \multicolumn{9}{|c|}{ 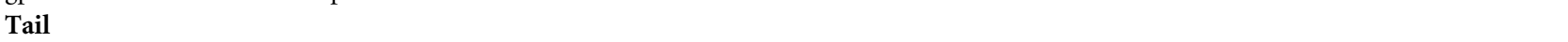 } \\
\hline gp10 & Baseplate wedge subunit & 10 & - & - & 18 & - & 66232 & - \\
\hline gp11 & Baseplate wedge subunit & 3 & - & - & 18 & - & 23707 & - \\
\hline gp18 & Contractile tail sheath protein & 6 & 18 & - & 138 & 102 & 71331 & 78689 \\
\hline gp19 & Tail tube protein & 3 & 3 & - & 144 & 103 & 18450 & 22790 \\
\hline gp25 & Baseplate wedge subunit & - & - & - & 6 & - & - & - \\
\hline gp26 & Baseplate hub subunit & - & - & - & & - & - & - \\
\hline gp3 & Tail completion protein & - & 2 & - & 6 & 110 & - & 19740 \\
\hline gp48 & Baseplate, tail-tube-associated & 1 & 2 & - & 6 & 200 & 39708 & 35302 \\
\hline gp5 & Baseplate hub subunit and tail lysozyme & 1 & - & - & 3 & - & 63116 & - \\
\hline gp53 & Baseplate wedge component & - & - & - & 6 & - & - & - \\
\hline gp6 & Baseplate wedge subunit & 3 & 3 & - & 12 & 80 & 74383 & 26031 \\
\hline gp7 & Baseplate wedge subunit & 14 & - & - & 6 & - & 119215 & - \\
\hline gp8 & Baseplate wedge subunit & 2 & 19 & - & 12 & 83 & 37983 & 69588 \\
\hline gp15 & Proximal tail sheath stabilizer & 2 & 5 & - & 6 & 95 & 31557 & 30458 \\
\hline gp9 & Baseplate wedge tail-fibre connector & 2 & - & - & 18 & - & 30977 & - \\
\hline gp29 & $\begin{array}{l}\text { Baseplate hub subunit, tail length } \\
\text { determinator }\end{array}$ & - & - & - & 6 & - & - & - \\
\hline gp27 & Baseplate hub subunit & 1 & - & - & 3 & - & 44360 & - \\
\hline gp28 & Baseplate hub distal subunit & - & - & - & & - & - & - \\
\hline \multicolumn{9}{|l|}{ Tail fibre } \\
\hline gp12 & Short tail fibre & 4 & - & - & $18 \dagger$ & - & 56214 & - \\
\hline gp34 & Long-tail-fibre component & 16 & - & - & $18 \dagger$ & - & 140403 & - \\
\hline gp36 & Long-tail-fibre component & 1 & - & - & $18 \dagger$ & - & 23342 & - \\
\hline gp37 & Tail-fibre component & 7 & - & - & $18 \dagger$ & - & 109224 & - \\
\hline gp35 & $\begin{array}{l}\text { Hinge connector of long tail fibre, } \\
\text { proximal connector }\end{array}$ & - & - & - & $6 \dagger$ & - & - & - \\
\hline \multicolumn{9}{|c|}{ Novel S-PM2 proteins } \\
\hline 82 & Unknown & - & 3 & & & 82 & & 19235 \\
\hline 86 & Unknown & - & 9 & & & 86 & & 134302 \\
\hline
\end{tabular}


Table 1. cont.

\begin{tabular}{|c|c|c|c|c|c|c|c|c|}
\hline $\begin{array}{l}\text { T4 gene } \\
\text { product }\end{array}$ & Function & \multicolumn{2}{|c|}{ No. of peptides } & \multicolumn{2}{|c|}{ Copy no.* } & S-PM2 ORF no. & \multicolumn{2}{|c|}{$\begin{array}{l}\text { Molecular mass } \\
\text { (Da) }\end{array}$} \\
\hline 87 & Unknown & - & 2 & & & 87 & & 18668 \\
\hline 89 & Unknown & - & 5 & & & 89 & & 33768 \\
\hline 90 & Unknown & - & 2 & & & 90 & & 37256 \\
\hline 145 & Unknown & - & 5 & & & 145 & & 32829 \\
\hline 172 & Unknown & - & 2 & & & 172 & & 118232 \\
\hline 174 & Unknown & - & 5 & & & 174 & & 120951 \\
\hline 219 & Unknown & - & 2 & & & 219 & & 32340 \\
\hline 221 & Unknown & - & 3 & & & 221 & & 59748 \\
\hline 223 & Unknown & - & 3 & & & 223 & & 114570 \\
\hline
\end{tabular}

*Data from Leiman et al. (2003), Akhter et al. (2007) and Kostyuchenko et al. (2005).

$\dagger$ For the tail fibres the number reported is copy number multiplied by 6 since each virion has 6 fibres.

al., 1994). This is consistent with the observation that gp22 was detected from a band of approximately $19 \mathrm{kDa}$ (as opposed to the theoretical molecular mass of $43.8 \mathrm{kDa}$ ).

In T4, gp20 is a dodecamer protein that forms the proximal vertex of the head and through which DNA is injected (Leiman et al., 2004). This protein was found in both S-PM2 and T4. gp13 forms a ring with gp14 that sits closest to the head (Akhter et al., 2007; Leiman et al., 2004). gp13 was detected in both S-PM2 and T4 preparations, but gp14 was not found in either phage preparation and thus it may not be amenable to the approach taken in this study (see discussion below). There is no homologue of the gene that encodes the whiskers (gpwac) in S-PM2, and electron microscopy studies have not revealed such a structure (Fig. 2).

\section{Tail sheath and tail tube}

The contractile tail sheath protein gp18 and tail tube protein gp19 are very abundant within the phage particle (Leiman et al., 2003). This abundance is consistent with their detection in both T4 and S-PM2 preparations. gp3 and gp 15 are at the top of the tail tube in T4 (Leiman et al., 2004). Although they are present at relatively low abundance, both proteins were found in S-PM2, and gp15 was detected in T4. The end of the tail tube in T4 is thought to be either gp48 or gp54; SPM2 only has homology with gp48, and this protein was detected in S-PM2.

\section{Baseplate}

At the bottom of the tail in T4 is a baseplate wedge which consists of gp6, gp7, gp8, gp10, gp11, gp25 and gp53. Six wedges are joined around the central hub with the help of gp9 (the long-tail-fibre attachment protein) and gp12 (the short tail fibre) (Kostyuchenko et al., 2003; Van Raaij et al., 2001). The hub consists of gp5, gp27 and gp29. These proteins in T4 were not amenable to our approach for identification and thus we detected only one peptide for gp5 and gp27 and none for gp29.

S-PM2 has homology to four of the baseplate wedge proteins (gp6, gp8, gp25 and gp53) and the hub protein and gp5. If the tail is relatively constrained in evolutionary terms, it seems likely that the proteins attached to it are also constrained. Of the five predicted baseplate proteins, only gp6 and gp8 were detected.

The baseplate-related ORFs present in the T4 genome are at either end of the structural gene cluster with the genes encoding gp5, gp6, gp7, gp8, gp9, gp10, gp11, gp12 and gp53 at one end, and the rest (gp25, gp26, gp27, gp28, gp29, gp48, gp51 and gp54) at the other (Miller et al., 2003a). This is not the case in other phages such as KVP40, where only one such cluster of baseplate proteins has been identified and this contains most of the genes found in the two separate clusters in T4 (Miller et al., 2003b). The arrangement in the S-PM2 genome seems to be similar to KVP40 in that the gene encoding gp6 is next to the gene encoding gp25. In this region of the S-PM2 genome there are several newly identified ORFs and it seems likely that some of these encode proteins involved in the baseplate. A second cluster of genes encoding baseplate subunits (gp53, gp48, gp26 and gp5) are found at around $150-160 \mathrm{~kb}$ of the genome, thus the two clusters of baseplate-related ORFs are not grouped in the same way in S-PM2 as they are in T4.

\section{Tail fibres}

The tail fibres are very important determinants of specificity in host-phage interactions. Unsurprisingly, due to the differences in the cyanobacterial and E. coli surfaces, the tail fibres of S-PM2 are highly divergent from known T4-type phages, with none of the predicted ORFs in S-PM2 resembling known tail fibres. Indeed, no tail fibres from 

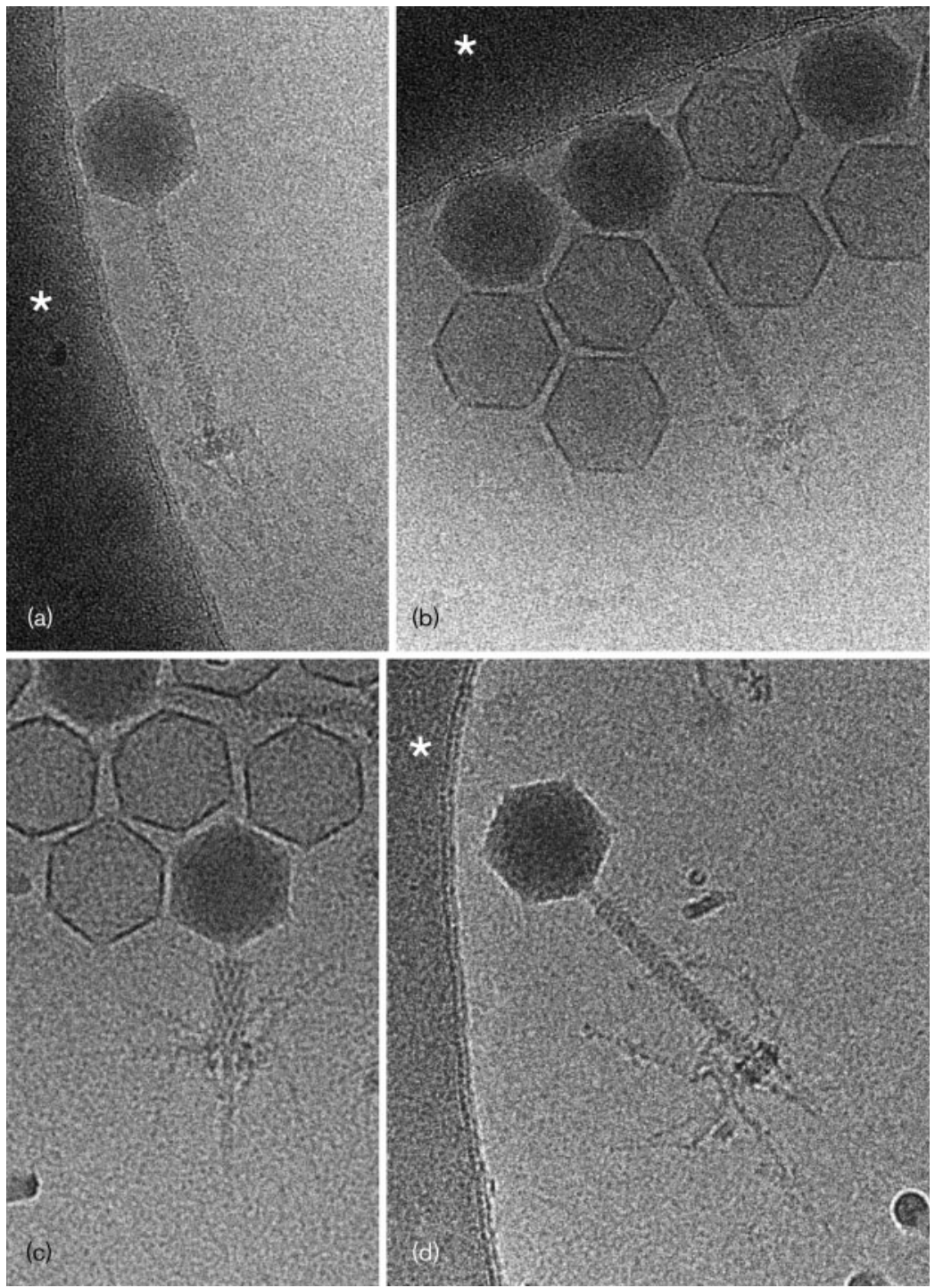

Fig. 2. Cryoelectron micrographs of purified S-PM2 phage. $(a, b)$ Lower value of underfocus (less contrast), showing the fine structure of the helical tails and DNA packing. The short tails are also visible in (a) just underneath the baseplate. (c) An S-PM2 phage with a contracted tail sheath. The lack of collar structure is particularly visible in (d). The diameter of the head is $65 \mathrm{~nm}$. The * symbol indicates the edge of the carbon, which delineates the hole where the phage sample is suspended in amorphous ice.

cyanophages have been identified by anything other than a bioinformatic approach.

In $\mathrm{T} 4$, the short tail fibres (gp12) recognize the bacterial cell surface and undergo an irreversible binding reaction (Miller et al., 2003a). Putative short tail fibres in S-PM2 may be encoded by ORF 225 which has two amino acid sequence domains which are similar to the tail-fibre receptor of T4 (gp12, with a BLAST e-value of $\left.6 \mathrm{e}^{-06}\right)$.

In T4, the long tail fibres are gp34, gp35, gp36 and gp37. Three of these - gp34, gp36 and gp37 - were detected in this study. Based on the fact that we found the T4 tail fibres, it seems likely that some of our 'new' proteins identified in this study are S-PM2 tail-fibre proteins. In T4 the only structural genes which are separated from all the other structural ORFs are the long-tail-fibre genes. Possible candidates for these tail-fibre genes in the S-PM2 genome are newly identified ORFs 172 and 174 that belong to a similarly discrete cluster.
In summary, a combination of proteomic and bioinformatic approaches has proved highly successful in the identification of proteins from the newly characterized virus S-PM2. At least $50 \%$ of the structural proteins from T4 and S-PM2 have been identified, including a significant number of head, tail, neck, baseplate and tail-fibre proteins. Other recent approaches based on N-terminal sequencing and on MS/MS have identified a total of 10 and 8 proteins, respectively (which assuming there are a similar number of proteins as there are in T4, is around 26 and $20 \%$ of total proteins in the virions, respectively), so our approach has been far more extensive in terms of coverage (Pope et al., 2007; Weigele et al., 2007). We are also the first to use this approach on T4, as a control, to see how many capsid proteins could be identified in a wellcharacterized bacteriophage.

The proteins that remained elusive T4 included those from the baseplate and head. This may be due to incompatibility 

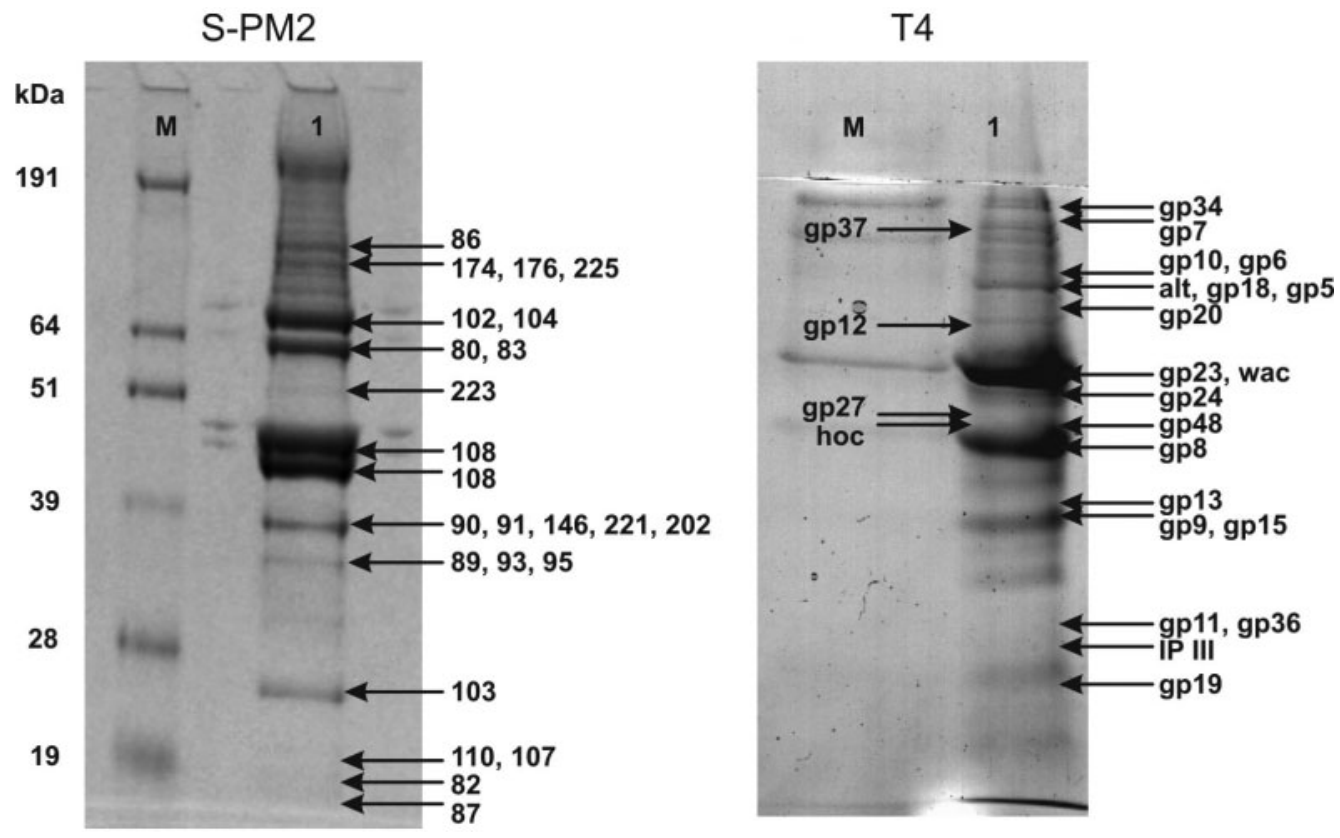

Fig. 3. S-PM2 and T4 structural proteins separated by 4-12\% SDS-PAGE. Lanes: M, protein standards; 1 S-PM2 (left-hand gel; ORF numbers are shown on the right) and T4 (right-hand gel). Samples were stained using Coomassie brilliant blue, excised and digested with trypsin, and analysed using LC-ESI-MS/MS.

of these proteins with our sample preparation procedures (e.g. a lack of solubility), or they may not be present in suitable abundance within the assembled phage capsid to be detected post-digestion in the mass spectrometer. Proteins present at very low concentrations may be masked by higher abundance proteins of similar molecular mass occurring in the same protein band. For example, in this study, gp23, gp18 and gp19 were highly abundant and therefore could mask proteins of a similar molecular mass. Furthermore, the presence of a protein on a gel does not ensure recovery of all the peptides following in-gel digestion (Speicher et al., 2000).

Certainly it seems that one or more of these underlying causes resulted in some proteins not being detected in either T4 or S-PM2. For example, the only three predicted proteins that we did not detect in S-PM2 (the neck protein gp14 and two of the baseplate proteins, gp25 and gp53) were also not detected in the T4 preparations. We propose to utilize alternate methodologies to identify the proteins that remain elusive to our gel-resolved sample preparation methods.

Our study clearly indicates widespread applicability and a high success rate of this approach in studying novel proteomes where little or no detectable homology with other organisms exists. The prerequisites of a study of this type are a genome sequence from which a proteome can be predicted, and in-house bioinformatics expertise to manipulate the various datasets produced in conjunction with MS-based protein identification techniques.

\section{REFERENCES}

Akhter, T., Zhao, L., Kohda, A., Mio, K., Kanamaru, S. \& Arisaka, F. (2007). The neck of bacteriophage T4 is a ring-like structure formed by a hetero-oligomer of gp13 and gp14. Biochim Biophys Acta 1774, 1036-1043.

Black, L. W., Showe, M. K. \& Steven, A. C. (1994). Morphogenesis of the T4 head. In Molecular Biology of Bacteriophage T4, pp. 218-258. Edited by J. D. Karam, J. W. Drake, K. N. Kreuzer and others. Washington, DC: American Society for Microbiology.

Field, C. B., Behrenfeld, M. J., Randerson, J. T. \& Falkowski, P. (1998). Primary production of the biosphere: integrating terrestrial and oceanic components. Science 281, 237-240.

Frank, J. (2006). Three Dimensional Electron Microscopy of Macromolecular Assemblies. Oxford: Oxford University Press.

Fuhrman, J. A. (1999). Marine viruses and their biogeochemical and ecological effects. Nature 399, 541-548.

Hambly, E., Tétart, F., Desplats, C., Wilson, W. H., Krisch, H. M. \& Mann, N. H. (2001). A conserved genetic module that encodes the major virion components in both the coliphage T4 and the marine cyanophage S-PM2. Proc Natl Acad Sci U S A 98, 11411-11416.

Konopa, G. \& Taylor, K. (1979). Coliphage lambda ghosts obtained by osmotic shock or $\mathrm{LiCl}$ treatment are devoid of $\mathrm{J}$ - and $\mathrm{H}$-gene products. J Gen Virol 43, 729-733.

Kostyuchenko, V. A., Leiman, P. G., Chipman, P. R., Kanamaru, S., van Raaij, M. J., Arisaka, F., Mesyanzhinov, V. V. \& Rossmann, M. (2003). Three-dimensional structure of bacteriophage T4 baseplate. Nat Struct Biol 10, 688-693.

Kostyuchenko, V. A., Chipman, P. R., Leiman, P. G., Arisaka, F., Mesyanzhinov, V. V. \& Rossmann, M. G. (2005). The tail structure of bacteriophage T4 and its mechanism of contraction. Nat Struct Mol Biol 12, 810-813. 
Leiman, P. G., Kanamaru, S., Mesyanzhinov, V. V., Arisaka, F. \& Rossmann, M. G. (2003). Structure and morphogenesis of bacteriophage T4. Cell Mol Life Sci 60, 2356-2370.

Leiman, P. G., Chipman, P. R., Kostyuchenko, V. A., Mesyanzhinov, V. V. \& Rossmann, M. G. (2004). Three-dimensional rearrangement of proteins in the tail of bacteriophage T4 on infection of its host. Cell 118, 419-429.

Mann, N. H., Clokie, M. R. J., Millard, A., Cook, A., Wilson, W. H., Wheatley, P. J., Letarov, A. \& Krisch, H. M. (2005). The genome of S-PM2, a 'photosynthetic' T4-type bacteriophage that infects marine Synechococcus strains. J Bacteriol 187, 3188-3200.

Miller, E. S., Kutter, E., Mosig, G., Arisaka, F., Kunisawa, T. \& Rüger, W. (2003a). Bacteriophage T4 genome. Microbiol Mol Biol Rev 67, 86-156.

Miller, E. S., Heidelberg, J. F., Eisen, J. A., Nelson, W. C., Durkin, A. S., Ciecko, A., Feldblyum, T. V., White, O., Paulsen, I. T. \& other authors (2003b). Complete genome sequence of the broad-host-range vibriophage KVP40: comparative genomics of a T4-related bacteriophage. J Bacteriol 185, 5220-5233.

Pope, W. H., Weigele, P. R., Chang, J., Pedulla, M. L., Ford, M. E., Houtz, J. M., Jiang, W., Chiu, W., Hatfull, G. F. \& other authors (2007). Genome sequence, structural proteins, and capsid organization of the cyanophage Syn5: a 'horned' bacteriophage of marine Synechococcus. J Mol Biol 368, 966-981.
Prangishvili, D. \& Garrett, R. J. (2004). Exceptionally diverse morphotypes and genomes of crenarchaeal hyperthermophilic viruses. Biochem Soc Trans 32, 204-208.

Sambrook, J., Fritsch, E. F. \& Maniatis, T. (1989). Molecular Cloning: a Laboratory Manual. Cold Spring Harbor, NY: Cold Spring Harbor Laboratory.

Speicher, K. D., Kolbas, O., Harper, S. \& Speicher, D. W. (2000). Systematic analysis of peptide recoveries from in-gel digestions for protein identifications in proteome studies. J Biomol Tech 11, 74-86.

Suttle, C. A. (2007). Marine viruses - major players in the global ecosystem. Nat Rev Microbiol 5, 801-812.

Van Raaij, M. J., Schoehn, G., Burda, M. R. \& Miller, S. (2001). Crystal structure of a heat and protease-stable part of the bacteriophage T4 short tail fibre. J Mol Biol 314, 1137-1146.

Weigele, P. R., Pope, W. H., Pedulla, M. L., Houtz, J. M., Smith, A. L., Conway, J. F., King, J., Hatfull, G. F., Lawrence, J. G. \& Hendrix, R. W. (2007). Genomic and structural analysis of Syn9, a cyanophage infecting marine Prochlorococcus and Synechococcus. Environ Microbiol 9, 1675-1695.

Wilson, W. H., Joint, I. R., Carr, N. G. \& Mann, N. H. (1993). Isolation and molecular characterization of 5 marine cyanophages propagated on Synechococcus sp. strain WH7803. Appl Environ Microbiol 59, 3736-3743.

Edited by: L. S. Frost 